\title{
Aneurisma roto da zona fibrosa intervalvar mitro-aórtica por endocardite infecciosa secundária à artrite séptica em paciente pediátrico: relato de caso
}

\section{Ruptured mitro-aortic intervalvar fibrous zone aneurysm due to infectious endocarditis secondary to septic arthritis in a pediatric patient: case report}

Catarina Padilha Tinoco ${ }^{1}$, Camile Torres Andriani ${ }^{1}$, Pedro Lukas do Rêgo Aquino ${ }^{1}$, Gabriela Padilha Tinoco ${ }^{1}$, Maria Améllia do Rêgo Aquino ${ }^{2}$, Marcele Torres Andriani ${ }^{3}$, Catarina Vasconcelos Cavalcanti ${ }^{1}$, Dário Celestino Sobral Filho ${ }^{1}$ ${ }_{1}^{1}$ Pronto Socorro Cardiológico de Pernambuco, Universidade de Pernambuco, Recife, Brasil.

${ }^{2}$ Hospital das Clínicas, Universidade Federal de Pernambuco, Recife, Brasil

${ }^{3}$ Faculdade de Ciências Médicas da Paraíba, João Pessoa, Brasil

\section{Resumo}

O aneurisma da zona fibrosa mitro-aórtica é uma complicação pouco frequente, mas potencialmente fatal da endocardite infecciosa de válvula aórtica. As manifestações clínicas desse aneurisma são bastante variáveis, podendo ser assintomáticas ou apresentar dor torácica, palpitações, sopros e disfunção mitral como sintomas. O diagnóstico é realizado principalmente pelo ecocardiograma transtorácico e transesofágico, no qual se evidencia uma cavidade perivalvular pulsátil com expansão sistólica e colapso diastólico localizada na zona mitro-aórtica. O tratamento recomendado para esses pacientes é a correção cirúrgica mesmo nos assintomáticos. Neste relato é apresentado o caso de uma criança de 10 anos, que desenvolveu um aneurisma da zona fibrosa intervalvar mitro-aórtica como uma complicação de um quadro de endocardite infecciosa secundária à artrite séptica.

Palavras chave: Aneurisma da fibrosa intervalvar mitro-aórtica, Endocardite, Artrite séptica.

\begin{abstract}
Mitral aortic fibrous aneurysm is an uncommon but potentially fatal complication of aortic valve infective endocarditis (IE). The clinical manifestations of this aneurysm are quite variable and may be asymptomatic or present chest pain, palpitations, murmurs and mitral dysfunction as symptoms. The diagnosis is mainly made by transthoracic and transesophageal echocardiography, which shows a pulsatile perivalvular cavity with systolic expansion and diastolic collapse located in the mitro-aortic zone. The recommended treatment for these patients is surgical correction even in asymptomatic patients. This report presents the case of a 10year-old child who developed a mitro-aortic intervalvar fibrous aneurysm as a complication of infectious endocarditis secondary to septic arthritis.
\end{abstract}

Keywords: Mitroaortic intervalvar fibrous aneurysm, Endocarditis, Septic arthritis.

\section{Introdução}

$\mathrm{O}$

aneurisma

da

zona

fibrosa intervalvar mitro-aórtica é raro, pode ser secundário a endocardite infecciosa, trauma torácico fechado, defeito congênito e complicação de cirurgia cardíaca. Pode ser assintomático ou apresentar dor torácica, palpitações, sopros e disfunção mitral como manifestações clínicas. ${ }^{1}$ Além disso, pode evoluir com complicações graves, como rotura para o pericárdio, aorta ou átrio esquerdo, compressão sistólica das artérias coronárias e insuficiência mitral (IM).

Neste relato, é descrito um caso de um aneurisma da fibrosa intervalvar mitro-aórtica como 
complicação de endocardite infecciosa secundária a artrite séptica após trauma em joelho esquerdo em paciente de 10 anos.

\section{Relato de Caso}

Paciente com 10 anos de idade, história de trauma fechado no membro inferior esquerdo, evoluiu com febre e secreção purulenta do joelho e dificuldade na deambulação. Ao exame físico apresentou perda ponderal acentuada, ritmo cardíaco regular com sopro sistólico regurgitativo em foco mitral e edema em joelho esquerdo. Demais sistemas sem alterações.

Foi encaminhado a serviço especializado, passou por cirurgia ortopédica e foi submetido a um ecocardiograma transesofágico, evidenciando vegetação de valva mitral de 7x3 mm e dilatação de átrio esquerdo e IM de moderada à grave.

Evoluiu com gravidade, necessitando suporte em terapia intensiva. Fez hemocultura que evidenciou $S$. aureus meticilina-resistente, foi então tratado por 55 dias com vancomicina. No $40^{\circ}$ dia de tratamento com vancomicina, apresentou febre e novas culturas foram todas negativas. A febre foi atribuída à flebite em membro superior.

Novo ecocardiograma, 4 semanas após o primeiro, evidenciou IM moderada por perfuração da base do folheto anterior e aneurisma roto da fibrosa intervalvar mitro-aórtica. $\mathrm{O}$ aneurisma foi observado se preenchendo durante a sístole, esvaziando-se durante a diástole e exibindo um fluxo em turbilhão. Foi solicitada angiotomografia, que confirmou o diagnóstico de aneurisma da fibrosa intervalvar mitro-aórtica.

Foi então realizada cirurgia para correção do aneurisma por aortotomia e plastia mitral com patch de pericárdio bovino. Durante a cirurgia não foi encontrado aspecto de endocardite na válvula. Fez uso de hemoderivados (uma unidade de concentrado de hemácias e duas unidades de plasma fresco congelado). O pós-operatório cursou sem intercorrências.

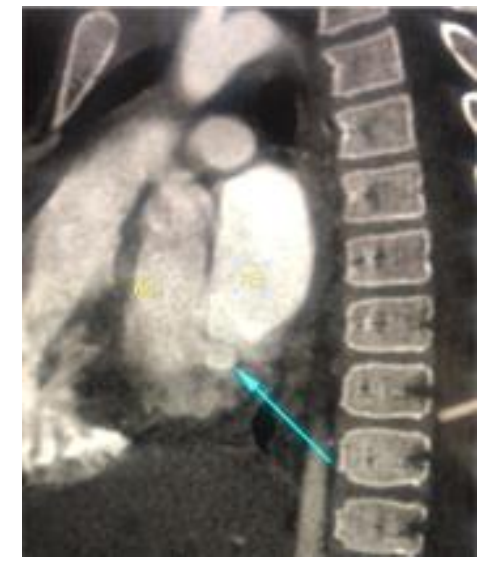

Figura 1. Imagem da angiotomografia computadorizada, que mostra (seta) aneurisma da fibrosa intervalvar mitro-aórtica

\section{Discussão}

Os aneurismas ventriculares subvalvares são lesões cardíacas pouco frequentes na prática clínica. Esses aneurismas são classificados em submitral e subaórtico. ${ }^{1}$ Os aneurismas submitrais são mais comuns e maiores que os subaórticos, bem como contêm trombos em sua cavidade, apresentam um colo maior e, na maioria dos casos, se relacionam com a cúspide posterior da valva mitral. $\mathrm{O}$ paciente relatado apresentou aneurisma subaórtico, o qual é menos frequente, geralmente é menor que o submitral e seu colo se relaciona, muitas vezes, com a membrana fibrosa localizada entre o folheto anterior da valva mitral e a válvula da valva aórtica fibrosa intervalvar mitro-aórtica (FIMA), ${ }^{2,3}$

A FIMA é uma estrutura fibrosa delgada que conecta a porção posterior da raiz aórtica à base do folheto anterior da valva mitral. ${ }^{4}$ Além disso, a FIMA é uma região de menor vascularização e, portanto, mais susceptível a lesões, como abcesso, aneurisma e perfuração, por exemplo. ${ }^{4}$ Aneurismas nessas regiões possuem apresentações clínicas variadas podendo ser desde assintomáticos até apresentar sintomatologias muito associadas à sua rotura causando, por exemplo, dor torácica, palpitação, sopros, sinais de insuficiência cardíaca (IC), disfunção valvar mitral e aórtica, síndrome coronariana aguda, bloqueio atrioventricular, rotura com tamponamento cardíaco ou morte súbita. ${ }^{3,4}$ As principais causas para o aparecimento dessas lesões são congênita, por defeito no desenvolvimento entre a parede ventricular muscular e o anel fibroso valvar; infecciosa, principalmente por endocardite infecciosa da valva aórtica; e traumática, como póscirúrgico. ${ }^{4}$ Dos fatores causadores descritos, o que 
desencadeou o aneurisma no caso exposto foi a endocardite infecciosa (EI).

A EI tem uma incidência de 3 a 9 casos por 100 mil pessoas e é uma importante causa de morbimortalidade. A infecção frequentemente produz vegetações, que são estruturas compostas de plaquetas, fibrina e microrganismos infecciosos, que podem ser evidenciadas no ecocardiograma, como aconteceu no caso relatado. O diagnóstico é feito pelos critérios de Duke modificados. ${ }^{5}$ A presença de dois critérios maiores, um maior associado a três menores ou cinco menores isoladamente, é suficiente para o diagnóstico. O paciente preencheu os critérios diagnósticos de Duke para EI, visto que apresentou, 2 critérios maiores e 2 menores: 1 aparecimento de sopro sistólico regurgitativo; 2 ecocardiograma com sinais de endocardite: vegetação em valva mitral, pseudoaneurisma e moderada regurgitação valvar mitral; 3 hemocultura positiva para S. aureus; 4 - febre. As complicações cardíacas, como pseudoaneurisma ventricular, abscesso, formação de fístula, embolia sistêmica e insuficiência cardíaca, destacam-se entre as principais causas de óbito, especialmente quando de evolução aguda, em pós-operatório. ${ }^{6} \mathrm{O}$ tratamento clínico da EI se baseia, sobretudo, na antibioticoterapia de acordo com o agente infeccioso isolado na hemocultura. Desse modo, como o agente infeccioso isolado no paciente descrito foi $o$ estafilococo aureus meticilina-resistente, foi optado por vancomicina com base na literatura. ${ }^{5,7}$ Além disso, o paciente relatado apresentou sinais de IC descompensada perfil $\mathrm{B}$, sendo iniciada terapia de suporte com furosemida, captopril e digitálicodigoxina. ${ }^{8,9}$

O diagnóstico do aneurisma pode ser feito através de ecocardiografia transtorácica e transesofágica, cateterismo cardíaco ou tomografia computadorizada, sendo o primeiro o método mais comum. O principal diagnóstico diferencial do aneurisma da fibrosa intervalvar mitro-aórtica é o abscesso do anel aórtico, porém no primeiro é evidenciado uma cavidade perivalvar pulsátil com expansão sistólica e colapso diastólico localizado na zona mitro-aórtica. ${ }^{10,12} \mathrm{O}$ paciente do caso teve o diagnóstico inicial pela ecocardiografia transtorácica, que foi posteriormente confirmado pela angiotomografia computadorizada. A rotura do aneurisma é um importante fator de risco para mortalidade nesses pacientes e foi evidenciada no caso relatado.
Para tratamento do pseudoaneurisma da fibrosa mitro-aórtica, a correção cirúrgica é a opção mais frequentemente empregada, mesmo em pacientes assintomáticos. ${ }^{13}$ No relato descrito, a perfuração do aneurisma com shunt da via de saída do ventrículo esquerdo para o átrio esquerdo, infecção, rotura do aneurisma e disfunções valvares fizeram com que a cirurgia fosse o tratamento mais efetivo. $\mathrm{O}$ tratamento cirúrgico consiste no reparo primário da válva aórtica e clampeamento do aneurisma ou no fechamento do colo com retalho de tecido. Em muitos casos é necessária a realização de troca valvar se houver infecção ou impossibilidade da sua reconstrução. Como a valva aórtica frequentemente está envolvida, a via de acesso de preferência é por aortotomia e, por esse motivo, foi a via de acesso optada nesse caso. A plastia mitral foi indicada nesse paciente devido a insuficiência mitral de grau moderado por perfuração da base do folheto anterior e realizado um patch de pericárdio bovino. ${ }^{14}$

\section{Conclusão}

Foi descrito caso de EI evoluindo com um aneurisma da fibrosa intervalvar mitro-aórtica e corrigido com sucesso por uma plastia mitral associada. A indicação cirúrgica para correção do aneurisma está atrelada ao surgimento de complicações, como perfuração do aneurisma com shunt entre ventrículo e átrio esquerdos, infecção, compressão das artérias coronárias ou pulmonares, aumento do aneurisma, com possibilidade de rotura, embolizações, disfunções valvares primárias ou decorrentes do aneurisma. Sendo assim, no caso descrito, a cirurgia estava indicada tanto pela IM, quanto pela perfuração do folheto anterior.

\section{Referências}

1. Bueno RM, Ávila Neto V, Melo RFA (1998) Correção cirúrgica com sucesso de aneurisma subaórtico: relato de caso. Bras Cir Cardiovasc 13(1):71-6.

2. Norman SJ (1991) Annular subaortic aneurysm resulting in sudden death. Clin Cardiol 14:67-72.

3. Lurie AO (1960) Left ventricular aneurysm in the African. Br Heart J 22: 181-8.

4. Karalis DG, Bansal RC, Hauck AJ, et al (1992) Transesophageal echocardiographic recognition of 
subaortic valve endocarditis. Circulation 86: 353 62.

5. Cheirlin MD, Alpert JS, Armstrong WE, et al (1997) ACC/AHA guidelines for the clinical application of echocardiography: executive sumary: a report of the American College of Cardiology/American Heart Association Task Force on Practice Guidelines (Committee on Clinical Application of Echocardiography). J Am Coll Cardiol 29:862-79.

6. Habib G, Hoen B, Tornos P, Thuny F, Prendergast B, Vilacosta I, et al (2009) Guidelines on the prevention, diagnosis and treatment of infective endocarditis new version. European Heart Journal 30:2369-2413.

7. Gould FK, Elliot TSJ, Foweraker J, Fulford M, Perry JD, Roberts GJ, et al (2012) Guidelines for the diagnosis ant antibiotic treatment of endocarditis in adults: a report of the Working Party of the British Society for Antimicrobial Chemotherapy. J Antimicrob Chemother 67:269-289.

8. Miró Ò, Hazlitt M, Escalda X, Llorens P, Gil V, Martín-Sánchez FJ, et al (2018) Effects of the intensity of prehospital treatment on short-term outcomes in patients with acute heart feilure: th e SEMICA-2 study. Clin Res Cardiol 107(4):347-61.

9. Testani JM, Chen J, McCauley BD, Kimmel SE, Shannon RP (2010) Potential effects of aggressive decongestion during the treatment of decompensated hert failure on renal functional and survival. Circulation 122(3):265-72.

10. Takimoto K, Arai F, Kita T, Sasaki S (2010) Pseudoaneurysm of the mitral aortic intervalvular fibrosa on a native aortic valve following infective endocarditis. J Anesth 24:260-3.

11. Mohan JC, Ali Khan J, Sethi KK, Arora R, Khalilullah M (1993) Two dimensional echocardiographic diagnosis of subaortic aneurysm. Indian Heart J 45:61-3.

12. Meyerowitz CB, Jacobs IE, Kotler MN, Loli AW, Wertheimer JH (1991) Four-year follow-up of a pseudoaneurysm of the mitral-aortic fibrosa. Am Heart J 122:589-92.

13. Head HD, Jue KL, Askren CC (1993) Aortic subannular ventricular aneurysms. ANN Thorac Surg 55: 1268-72.

14. Bansal RC, Graham BM, Juyzy KR, Shakudo M, Shah P (1990) Left ventricular outflow tract to left atrial communication secondary to rupture of mitral-aortic intervalvuvar fibrosa in infectibe endocarditis: diagnosis by transesophageal echocardiography and color flow imaging. J Am Coll Cardiol 15:499-504.

Autor para correspondência:

Catarina Padilha Tinoco : catarinaptinoco@hotmail.com

Recebido: 25/12/2019

Aceito: 07/02/2020 